\title{
Dual-Complex Jacobsthal Quaternions
}

\author{
Fügen Torunbalcı Aydın*
}

\begin{abstract}
In this paper, dual-complex Jacobsthal quaternions are defined. Also, some algebraic properties of dualcomplex Jacobsthal quaternions which are connected with dual-complex numbers and Lucas numbers are investigated. Furthermore, the Honsberger identity, the d'Ocagne's identity, Binet's formula, Cassini's identity, Catalan's identity for these quaternions and their real representations are given.
\end{abstract}

Keywords: Dual number; dual-complex number; Jacobsthal number; dual-complex Jacobsthal number; Jacobsthal quaternion; dual-complex Jacobsthal quaternion.

AMS Subject Classification (2020): Primary: 11B37; Secondary: 20G20; 11R52.

${ }^{*}$ Corresponding author

\section{Introduction}

The real quaternions were first described by Irish mathematician William Rowan Hamilton in 1843. Hamilton [1] introduced a set of real quaternions which can be represented as

$$
H=\left\{q=q_{0}+i q_{1}+j q_{2}+k q_{3} \mid q_{0}, q_{1}, q_{2}, q_{3} \in \mathbb{R}\right\}
$$

where

$$
i^{2}=j^{2}=k^{2}=-1, i j=-j i=k, \quad j k=-k j=i, \quad k i=-i k=j .
$$

The real quaternions constitute an extension of complex numbers into a four-dimensional space and can be considered as four-dimensional vectors, in the same way that complex numbers are considered as two-dimensional vectors.

In 1973, the first use of this numbers appears "A Handbook of Integer Sequences" in a paper by Sloane by the title "applications of Jacobsthal sequences to curves" [2]. In 1996, Horadam [3] introduced Jacobsthal and Jacobsthal-Lucas representation numbers. In 1997, Horadam [4], defined Jacobsthal representation polynomials. Several authors worked on Jacobsthal numbers by using Binet formulae or matrix method [12, 13, 16-19]. In 2016, Szynal-Liana and Wloch [20] defined Jacobsthal and Jacobsthal-Lucas quaternions respectively as follows

$$
J Q_{n}=J_{n}+i J_{n+1}+j J_{n+2}+k J_{n+3}
$$

and

$$
J L Q_{n}=j_{n}+i j_{n+1}+j j_{n+2}+k j_{n+3}
$$

where $J_{n}$ and $j_{n}$ denote the $n t h$ Jacobsthal and Jacobsthal-Lucas numbers, respectively. Also, the imaginary quaternion units $i, j, k$ have the following rules

$$
i^{2}=j^{2}=k^{2}=-1, i j=-j i=k, \quad j k=-k j=i, \quad k i=-i k=j
$$

Received : 17-09-2019, Accepted : 08-10-2020 
In 2017, Torunbalc1 Aydın and Yüce [21] given a new approach to Jacobsthal quaternions.

In 2017, Taşçı [22] defined k-Jacobsthal and Jacobsthal-Lucas quaternions as follows

$$
\begin{gathered}
Q J_{k, n}=J_{k, n}+i J_{k, n+1}+j J_{k, n+2}+k J_{k, n+3} \\
i^{2}=j^{2}=k^{2}=-1
\end{gathered}
$$

In 2020, Torunbalc1 Aydın [23] defined dual Jacobsthal quaternions as follows

$$
J_{D}=\left\{D_{n}^{J}=J_{n}+i J_{n+1}+j J_{n+2}+k J_{n+3} \mid J_{n} n-t h \text { Jacobsthal number }\right\},
$$

where

$$
i^{2}=j^{2}=k^{2}=i j k=0, i j=-j i=j k=-k j=k i=-i k=0 .
$$

Also, the dual Jacobsthal-Lucas quaternion can be defined as follows:

\begin{tabular}{|c|c|c|}
\hline & Definition & Multiplication Rules \\
\hline Jacobsthal quaternions & $\begin{array}{c}J Q_{n}=\left(J_{n}, J_{n+1}, J_{n+2}, J_{n+3}\right) \\
J_{n}=J_{n-1}+2 J_{n-2}, J_{1}=J_{2}=1\end{array}$ & $\begin{array}{c}(1, i, j, k), i^{2}=j^{2}=k^{2}=-1 \\
i j=-j i=k, j k=-k j=i, k i=-i k=j\end{array}$ \\
\hline $\mathrm{k}$-Jacobsthal quaternions & $\begin{array}{c}Q J_{k, n}=\left(J_{k, n}, J_{k, n+1}, J_{k, n+2}, J_{k, n+3}\right) \\
Q J_{k, n+2}=k Q J_{k, n+1}+2 Q J_{k, n}\end{array}$ & $\left(1, i_{1}, i_{2}, i_{3}\right), i_{1}^{2}=i_{2}^{2}=i_{3}^{2}=i_{1} i_{2} i_{3}=-1$ \\
\hline Dual Jacobsthal quaternions & $\begin{array}{c}D_{n}^{J}=\left(J_{n}, J_{n+1}, J_{n+2}, J_{n+3}\right) \\
J_{n}=J_{n_{1}}+2 J_{n-2}, J_{1}=J_{2}=1\end{array}$ & $\begin{array}{c}(1, i, j, k) i^{2}=j^{2}=k^{2}=i j k=0 \\
i j=-j i=j k=-k j=k i=-i k=0\end{array}$ \\
\hline Dual-complex Jacobsthal quaternions & $\begin{array}{c}Q_{J_{n}}=\left(J_{n}, J_{n+1}, J_{n+2}, J_{n+3}\right) \\
J_{n}=J_{n-1}+2 J_{n-2}, J_{1}=J_{2}=1\end{array}$ & $\begin{array}{c}(1, i, \varepsilon, i \varepsilon), i^{2}=-1, \varepsilon^{2}=0, \varepsilon \neq 0 \\
(i \varepsilon)^{2}=0\end{array}$ \\
\hline
\end{tabular}

$$
\begin{gathered}
j_{D}=\left\{D_{n}^{j}=j_{n}+i j_{n+1}+j j_{n+2}+k j_{n+3} \mid j_{n} n-t h \text { Jacobsthal-Lucas number }\right\}, \\
i^{2}=j^{2}=k^{2}=i j k=0, i j=-j i=j k=-k j=k i=-i k=0 .
\end{gathered}
$$

All the studies on Jacobsthal quaternions are summarized in Table 1.

Table 1. Types of Jacobsthal quaternions [20-23].

In the 19 th century Clifford invented a new number system by using the notation $(\varepsilon)^{2}=0, \varepsilon \neq 0$. This number system was called dual number system and the dual numbers were represented in the form $A=a+\varepsilon a^{*}$ with $a, a^{*} \in \mathbb{R}$ [5]. Afterwards, Kotelnikov (1895) and Study (1903) generalized first applications of dual numbers to mechanics [7], [8]. Besides mechanics, this concept has lots of applications in different areas such as algebraic geometry, kinematics, quaternionic formulation of motion in the theory of relativity. Majernik has introduced the multicomponent number system [9]. There are three types of the four-component number systems which have been constructed by joining the complex, binary and dual two-component numbers. Later, Farid Messelmi has defined the algebraic properties of the dual-complex numbers in the light of this study [10].There are many applications for the theory of dual-complex numbers. In 2017, [11] has defined the dual-complex Fibonacci numbers.

Dual-complex numbers [10] $w$ can be expressed in the form as

$$
\mathbb{D} \mathbb{C}=\left\{w=z_{1}+\varepsilon z_{2} \mid z_{1}, z_{2} \in \mathbb{C} \text { where } \varepsilon^{2}=0, \varepsilon \neq 0\right\} .
$$

Here if $z_{1}=x_{1}+i x_{2}$ and $z_{2}=y_{1}+i y_{2}$, then any dual-complex number can be written

$$
\begin{gathered}
w=x_{1}+i x_{2}+\varepsilon y_{1}+i \varepsilon y_{2} \\
i^{2}=-1, \quad \varepsilon \neq 0, \varepsilon^{2}=0, \quad(i \varepsilon)^{2}=0 .
\end{gathered}
$$

Addition, substraction and multiplication of any two dual-complex numbers $w_{1}$ and $w_{2}$ are defined by

$$
\begin{aligned}
w_{1} \pm w_{2} & =\left(z_{1}+\varepsilon z_{2}\right) \pm\left(z_{3}+\varepsilon z_{4}\right)=\left(z_{1} \pm z_{3}\right)+\varepsilon\left(z_{2} \pm z_{4}\right), \\
w_{1} \times w_{2} & =\left(z_{1}+\varepsilon z_{2}\right) \times\left(z_{3}+\varepsilon z_{4}\right)=z_{1} z_{3}+\varepsilon\left(z_{2} z_{4}+z_{2} z_{3}\right) .
\end{aligned}
$$


On the other hand, the division of two dual-complex numbers are given by

$$
\begin{aligned}
& \frac{w_{1}}{w_{2}}=\frac{z_{1}+\varepsilon z_{2}}{z_{3}+\varepsilon z_{4}} \\
& \frac{\left(z_{1}+\varepsilon z_{2}\right)\left(z_{3}-\varepsilon z_{4}\right)}{\left(z_{3}+\varepsilon z_{4}\right)\left(z_{3}-\varepsilon z_{4}\right)}=\frac{z_{1}}{z_{3}}+\varepsilon \frac{z_{2} z_{3}-z_{1} z_{4}}{z_{3}^{2}} .
\end{aligned}
$$

If $\operatorname{Re}\left(w_{2}\right) \neq 0$, then the division $\frac{w_{1}}{w_{2}}$ is possible. The dual-complex numbers are defined by the basis $\{1, i, \varepsilon, i \varepsilon\}$. Therefore, dual-complex numbers, just like quaternions, are a generalization of complex numbers by means of entities specified by four-component numbers. But real and dual quaternions are non commutative, whereas, dual-complex numbers are commutative. The real and dual quaternions form a division algebra, but dual-complex numbers form a commutative ring with characteristics 0 . Moreover, the multiplication of these numbers gives the dual-complex numbers the structure of 2-dimensional complex Clifford Algebra and 4-dimensional real Clifford Algebra. The base elements of the dual-complex numbers satisfy the following commutative multiplication scheme [10] (Table 2).

Table 2. Multiplication scheme of dual-complex numbers

\begin{tabular}{ccccc}
\hline $\mathrm{x}$ & 1 & $i$ & $\varepsilon$ & $i \varepsilon$ \\
\hline 1 & 1 & $i$ & $\varepsilon$ & $i \varepsilon$ \\
$i$ & $i$ & -1 & $i \varepsilon$ & $-\varepsilon$ \\
$\varepsilon$ & $\varepsilon$ & $i \varepsilon$ & 0 & 0 \\
$i \varepsilon$ & $i \varepsilon$ & $-\varepsilon$ & 0 & 0 \\
\hline
\end{tabular}

Five different conjugations can operate on dual-complex numbers [10] as follows:

$$
\begin{aligned}
w & =x_{1}+i x_{2}+\varepsilon y_{1}+i \varepsilon y_{2} \\
w^{*_{1}} & =\left(x_{1}-i x_{2}\right)+\varepsilon\left(y_{1}-i y_{2}\right)=\left(z_{1}\right)^{*}+\varepsilon\left(z_{2}\right)^{*}, \\
w^{*_{2}} & =\left(x_{1}+i x_{2}\right)-\varepsilon\left(y_{1}+i y_{2}\right)=z_{1}-\varepsilon z_{2}, \\
w^{*_{3}} & =\left(x_{1}-i x_{2}\right)-\varepsilon\left(y_{1}-i y_{2}\right)=z_{1}^{*}-\varepsilon z_{2}^{*}, \\
w^{*_{4}} & =\left(x_{1}-i x_{2}\right)\left(1-\varepsilon \frac{y_{1}+i y_{2}}{x_{1}+i x_{2}}\right)=\left(z_{1}\right)^{*}\left(1-\varepsilon \frac{z_{2}}{z_{1}}\right), \\
w^{*_{5}} & =\left(y_{1}+i y_{2}\right)-\varepsilon\left(x_{1}-i x_{2}\right)=z_{2}-\varepsilon z_{1} .
\end{aligned}
$$

Therefore, the norm of the dual-complex numbers is defined as

$$
\begin{aligned}
& N_{w}^{*_{1}}=\left\|w \times w^{*_{1}}\right\|=\sqrt{\left|z_{1}\right|^{2}+2 \varepsilon \operatorname{Re}\left(z_{1} z_{2}{ }^{*}\right)}, \\
& N_{w}^{*_{2}}=\left\|w \times w^{*_{2}}\right\|=\sqrt{z_{1}^{2}} \\
& N_{w}^{*_{3}}=\left\|w \times w^{*_{3}}\right\|=\sqrt{\left|z_{1}\right|^{2}-2 i \varepsilon \operatorname{Im}\left(z_{1} z_{2}{ }^{2}\right)}, \\
& N_{w}^{*_{4}}=\left\|w \times w^{*_{4}}\right\|=\sqrt{\left|z_{1}\right|^{2}} \\
& N_{w}^{*_{5}}=\left\|w \times w^{*_{5}}\right\|=\sqrt{z_{1} z_{2}+\varepsilon\left(z_{2}^{2}-z_{1}^{2}\right)} .
\end{aligned}
$$

In 2017, the dual-complex Fibonacci and Lucas numbers defined by Güngör and Azak [11] with the basis $\{1, i, \varepsilon, i \varepsilon\}$, where $i, \varepsilon$ and $i \varepsilon$ satisfy the conditions

$$
i^{2}=-1, \varepsilon \neq 0, \varepsilon^{2}=0, \quad(i \varepsilon)^{2}=0 .
$$

as follows

$$
\mathbb{D} \mathbb{C} F_{n}=\left(F_{n}+i F_{n+1}\right)+\varepsilon\left(F_{n+2}+i F_{n+3}\right)
$$


and

$$
\mathbb{D} \mathbb{C} L_{n}=\left(L_{n+i} L_{n+1}\right)+\varepsilon\left(L_{n+2}+i L_{n+3}\right) .
$$

In this paper, the dual-complex Jacobsthal numbers and quaternions will be defined. The aim of this work is to present in a unified manner a variety of algebraic properties of the dual-complex Jacobsthal quaternions as well as both the dual-complex numbers and dual-complex Jacobsthal numbers. In particular, using five types of conjugations, all the properties established for dual-complex numbers and dual-complex Jacobsthal numbers are also given for the dual-complex Jacobsthal quaternions. In addition, Binet's Formula, the Honsberger identity, the d'Ocagne's identity, Cassini's identity and Catalan's identity for these quaternions are given.

\section{The dual-complex Jacobsthal numbers}

In this paper, the dual-complex Jacobsthal and Jacobsthal-Lucas numbers defined by the basis $\{1, i, \varepsilon, i \varepsilon\}$, where $i, \varepsilon$ and $i \varepsilon$ satisfy the conditions

$$
i^{2}=-1, \quad \varepsilon \neq 0, \varepsilon^{2}=0, \quad(i \varepsilon)^{2}=0 .
$$

as follows

$$
\mathbb{D} \mathbb{C} J_{n}=J_{n}+i J_{n+1}+\varepsilon J_{n+2}+i \varepsilon J_{n+3}
$$

and

$$
\mathbb{D} C j_{n}=j_{n}+i j_{n+1}+\varepsilon j_{n+2}+i \varepsilon j_{n+3}
$$

With the addition and multiplication by real scalars of two dual-complex Jacobsthal numbers, the dual-complex Jacobsthal number can be obtained again. Then, the addition and subtraction of the dual-complex Jacobsthal numbers are defined by

$$
\begin{aligned}
\mathbb{D} \mathbb{C} J_{n} \pm \mathbb{D} \mathbb{C} J_{m}= & \left(J_{n} \pm J_{m}\right)+i\left(J_{n+1} \pm J_{m+1}\right)+\varepsilon\left(J_{n+2} \pm J_{m+2}\right) \\
& +i \varepsilon\left(J_{n+3} \pm J_{m+3}\right)
\end{aligned}
$$

The multiplication of a dual-complex Jacobsthal number by the real scalar $\lambda$ is defined as

$$
\lambda \mathbb{D} \mathbb{C} J_{n}=\lambda J_{n}+i \lambda J_{n+1}+\varepsilon \lambda J_{n+2}+i \varepsilon \lambda J_{n+3} .
$$

The multiplication of two dual-complex Jacobsthal numbers is defined by

$$
\begin{aligned}
\mathbb{D} \mathbb{C} J_{n} \times \mathbb{D} \mathbb{C} J_{m}= & \left(J_{n} J_{m}-J_{n+1} J_{m+1}\right)+i\left(J_{n+1} J_{m}+J_{n} J_{m+1}\right) \\
& +\varepsilon\left(J_{n} J_{m+2}-J_{n+1} J_{m+3}+J_{n+2} J_{m}-J_{n+3} J_{m+1}\right) \\
& +i \varepsilon\left(J_{n+1} J_{m+2}+J_{n} J_{m+3}+J_{n+3} J_{m}+J_{n+2} J_{m+1}\right) \\
= & \mathbb{D} \mathbb{C} J_{m} \times \mathbb{D} \mathbb{C} J_{n} .
\end{aligned}
$$

Also,the dual-complex Jacobsthal numbers provide the properties of Eq.(1.6)-(1.7) [10].

Five different conjugations can operate on dual-complex Jacobsthal numbers as follows:

$$
\begin{aligned}
& \mathbb{D} \mathbb{C} J_{n}{ }^{*_{1}}=\left(J_{n}-i J_{n+1}\right)+\varepsilon\left(J_{n+2}-i J_{n+3}\right), \\
& \mathbb{D} \mathbb{C} J_{n}{ }^{*_{2}}=\left(J_{n}+i J_{n+1}\right)-\varepsilon\left(J_{n+2}+i J_{n+3}\right), \\
& \mathbb{D} \mathbb{C} J_{n}{ }^{*_{3}}=\left(J_{n}-i J_{n+1}\right)-\varepsilon\left(J_{n+2}-i J_{n+3}\right), \\
& \mathbb{D} \mathbb{C} J_{n}{ }^{*_{4}}=\left(J_{n}-i J_{n+1}\right)\left(1-\varepsilon \frac{J_{n+2}+i J_{n+3}}{J_{n}+i J_{n+1}}\right), \\
& \mathbb{D C} J_{n}{ }^{*_{5}}=\left(J_{n+2}+i J_{n+3}\right)-\varepsilon\left(J_{n}+i J_{n+1}\right) .
\end{aligned}
$$


Therefore, the norm of the dual-complex Jacobsthal numbers is defined as

$$
\begin{aligned}
\left\|\mathbb{D} \mathbb{C} J_{n} \times \mathbb{D} \mathbb{C} J_{n}{ }^{*_{1}}\right\| & =\sqrt{\left|\left({J_{n}}^{2}+{J_{n+1}}^{2}\right)+2 \varepsilon\left(J_{n} J_{n+2}+J_{n+1} J_{n+3}\right)\right|} \\
& =\sqrt{\left|\left(J_{2 n+1}-J_{n}^{2}\right)+2 \varepsilon\left(3 J_{n} J_{n+1}+J_{2 n+1}+2 J_{n+1}^{2}\right)\right|}, \\
\left\|\mathbb{D} \mathbb{C} J_{n} \times \mathbb{D} \mathbb{C} J_{n}{ }^{*_{2}}\right\| & =\sqrt{\left|\left({J_{n}}^{2}-J_{n+1}{ }^{2}\right)+2 i J_{n} J_{n+1}\right|}, \\
\left\|\mathbb{D} \mathbb{C} J_{n} \times \mathbb{D} \mathbb{C} J_{n}{ }^{*_{3}}\right\| & =\sqrt{\left|J_{n}^{2}+J_{n+1}^{2}-2 i \varepsilon\left(J_{n+1} J_{n+2}-J_{n} J_{n+3}\right)\right|} \\
& =\sqrt{\left|J_{2 n+1}-J_{n}^{2}+2 i \varepsilon(-1)^{n} 2^{n}\right|}, \\
\left\|\mathbb{D} \mathbb{C} J_{n} \times \mathbb{D} \mathbb{C} J_{n}{ }^{*}\right\| & \sqrt{\left|{J_{n}}^{2}+J_{n+1}{ }^{2}\right|}=\sqrt{\left|J_{2 n+1}-J_{n}^{2}\right|} .
\end{aligned}
$$

\section{The dual-complex Jacobsthal quaternions}

In this section, firstly the dual-complex Jacobsthal quaternions will be defined. Later on, the definitions of the conjugations and norms of any dual-complex Jacobsthal number will be given.

The dual-complex Jacobsthal quaternions are defined by using the dual-complex numbers as follows

$$
\begin{gathered}
\mathbb{D C}^{J_{n}}=\left\{Q_{J_{n}}=J_{n}+i J_{n+1}+\varepsilon J_{n+2}+i \varepsilon J_{n+3} \mid J_{n}, \text { nth Jacobsthal number }\right\} \\
i^{2}=-1, \varepsilon \neq 0, \varepsilon^{2}=0, \quad(i \varepsilon)^{2}=0 .
\end{gathered}
$$

Let $Q_{J_{n}}$ and $Q_{J_{m}}$ be two dual-complex Jacobsthal quaternions such that

$$
Q_{J_{n}}=J_{n}+i J_{n+1}+\varepsilon J_{n+2}+i \varepsilon J_{n+3}
$$

and

$$
Q_{J_{m}}=J_{m}+i J_{m+1}+\varepsilon J_{m+2}+i \varepsilon J_{m+3} .
$$

Then, the addition and subtraction of two dual-complex Jacobsthal quaternions are defined in the obvious way,

$$
\begin{aligned}
Q_{J_{n}} \pm Q_{J_{m}}= & \left(J_{n}+i J_{n+1}+\varepsilon J_{n+2}+i \varepsilon J_{n+3}\right) \\
& \pm\left(J_{m}+i J_{m+1}+\varepsilon J_{m+2}+i \varepsilon J_{m+3}\right) \\
= & \left(J_{n} \pm J_{m}\right)+i\left(J_{n+1} \pm J_{m+1}\right)+\varepsilon\left(J_{n+2} \pm J_{m+2}\right) \\
& +i \varepsilon\left(J_{n+3} \pm J_{m+3}\right) .
\end{aligned}
$$

Multiplication of two dual-complex Jacobsthal quaternions is defined by

$$
\begin{aligned}
Q_{J_{n}} \times Q_{J_{m}}= & \left(J_{n}+i J_{n+1}+\varepsilon J_{n+2}+i \varepsilon J_{n+3}\right) \\
& \left(J_{m}+i J_{m+1}+\varepsilon J_{m+2}+i \varepsilon J_{m+3}\right) \\
= & \left(J_{n} J_{m}-J_{n+1} J_{m+1}\right)+i\left(J_{n+1} J_{m}+J_{n} J_{m+1}\right) \\
& +\varepsilon\left(J_{n} J_{m+2}-J_{n+1} J_{m+3}+J_{n+2} J_{m}-J_{n+3} J_{m+1}\right) \\
& +i \varepsilon\left(J_{n+1} J_{m+2}+J_{n} J_{m+3}+J_{n+3} J_{m}+J_{n+2} J_{m+1}\right) \\
= & Q_{J_{m}} \times Q_{J_{n}} .
\end{aligned}
$$

The scaler and the dual-complex vector parts of the dual-complex Jacobsthal quaternion $\left(Q_{J_{n}}\right)$ are denoted by

$$
S_{Q_{J_{n}}}=J_{n} \text { and } V_{Q_{J_{n}}}=i J_{n+1}+\varepsilon J_{n+2}+i \varepsilon J_{n+3} .
$$

Thus, the dual-complex Jacobsthal quaternion $Q_{J_{n}}$ is given by $Q_{J_{n}}=S_{Q_{J} n}+V_{Q_{J} n}$. Then, relation (3.5) is defined by

$$
\begin{aligned}
Q_{J_{n}} \times Q_{J_{m}} & =S_{Q_{J_{n}}} S_{Q_{J_{m}}}+S_{Q_{J_{n}}} V_{Q_{J_{m}}}+S_{Q_{J_{m}}} V_{Q_{J_{n}}}+V_{Q_{J_{n}}} \times V_{Q_{J_{m}}} \\
& =Q_{J_{m}} \times Q_{J_{n}} .
\end{aligned}
$$

The five kinds of conjugation given for the dual-complex Jacobsthal numbers are the same within the dualcomplex Jacobsthal quaternions. Furthermore, the conjugation properties for these quaternions are given by the relations in Eq.(2.6) as follows

$$
\left(Q_{J_{n}}\right)^{*_{1}}=J_{n}-i J_{n+1}+\varepsilon J_{n+2}-i \varepsilon J_{n+3}, \quad \text { complex - conjugation }
$$




$$
\begin{gathered}
\left(Q_{J_{n}}\right)^{*_{2}}=J_{n}+i J_{n+1}-\varepsilon J_{n+2}-i \varepsilon J_{n+3}, \text { dual - conjugation } \\
\left(Q_{J_{n}}\right)^{*_{3}}=J_{n}-i J_{n+1}-\varepsilon J_{n+2}+i \varepsilon J_{n+3}, \quad \text { coupled - conjugation } \\
\left(Q_{J_{n}}\right)^{*_{4}}=\left(J_{n}-i J_{n+1}\right) .\left(1-\varepsilon \frac{J_{n+2}+i J_{n+3}}{J_{n}+i J_{n+1}}\right), \quad \text { dual-complex - conjugation } \\
\left(Q_{J_{n}}\right)^{*_{5}}=J_{n+2}+i J_{n+3}-\varepsilon J_{n}-i \varepsilon J_{n+1}, \text { anti-dual-conjugation }
\end{gathered}
$$

Therefore, the norm of the dual-complex Jacobsthal quaternion $Q_{J_{n}}$ is defined as follows

$$
\begin{aligned}
\left.N_{(} Q_{J_{n}}\right)^{*_{1}}= & \left\|Q_{J_{n}} \times\left(Q_{J_{n}}\right)^{*_{1}}\right\|^{2} \\
= & \left|\left(J_{n}^{2}+J_{n+1}^{2}\right)+2 \varepsilon\left(J_{n} J_{n+2}+J_{n+1} J_{n+3}\right)\right| \\
= & \left|\left(J_{2 n+1}-J_{n}^{2}\right)+2 \varepsilon\left(3 J_{n} J_{n+1}+J_{2 n+1}+2 J_{n+1}^{2}\right)\right|, \\
\left.N_{(} Q_{J_{n}}\right)^{*_{2}}= & \left\|Q_{J_{n}} \times\left(Q_{J_{n}}\right)^{*_{2}}\right\|^{2}=\left|\left(J_{n}^{2}-J_{n+1}^{2}\right)+2 i J_{n} J_{n+1}\right|, \\
\left.N_{(} Q_{J_{n}}\right)^{*_{3}} & =\left\|Q_{J_{n}} \times\left(Q_{J_{n}}\right)^{*_{3}}\right\|^{2} \\
& =\left|\left(J_{n}^{2}+J_{n+1}^{2}\right)-2 i \varepsilon\left(J_{n+1} J_{n+2}-J_{n} J_{n+3}\right)\right| \\
& =\left|J_{2 n+1}-J_{n}^{2}+2 i \varepsilon(-1)^{n} 2^{n}\right| . \\
\left.N_{(} Q_{J_{n}}\right)^{*_{4}}= & \left\|Q_{J_{n}} \times\left(Q_{J_{n}}\right)^{*_{4}}\right\|^{2}=\left|J_{n}^{2}+J_{n+1}^{2}\right|=\left|J_{2 n+1}-J_{n}^{2}\right| .
\end{aligned}
$$

In the following theorem, some properties related to the conjugations of the dual-complex Jacobsthal quaternions are given.

Theorem 3.1. Let $\left(Q_{J_{n}}\right)^{*_{1}},\left(Q_{J_{n}}\right)^{*_{2}},\left(Q_{J_{n}}\right)^{*_{3}},\left(Q_{J_{n}}\right)^{*_{4}}$ and $\left(Q_{J_{n}}\right)^{*_{5}}$, be five kinds of conjugation of the dual-complex Jacobsthal quaternion. In this case, we can give the following relations:

$$
\begin{gathered}
Q_{J_{n}}\left(Q_{J_{n}}\right)^{*_{1}}=\left(J_{2 n+1}-J_{n}^{2}\right)+2 \varepsilon\left(3 J_{n} J_{n+1}+J_{2 n+1}+2 J_{n+1}^{2}\right), \\
Q_{J_{n}}\left(Q_{J_{n}}\right)^{*_{2}}=\left(J_{n}^{2}-J_{n+1}^{2}\right)-2 i J_{n} J_{n+1}, \\
Q_{J_{n}}\left(Q_{J_{n}}\right)^{*_{3}}=\left(J_{2 n+1}-J_{n}^{2}\right)-2 i \varepsilon(-1)^{n+1} 2^{n}, \\
Q_{J_{n}}\left(Q_{J_{n}}\right)^{*_{4}}=J_{2 n+1}-J_{n}^{2}, \\
Q_{J_{n}}+\left(Q_{J_{n}}\right)^{*_{1}}=2\left(J_{n}+\varepsilon J_{n+2}\right), \\
Q_{J_{n}}+\left(Q_{J_{n}}\right)^{*_{2}}=2\left(J_{n}+i J_{n+1}\right), \\
Q_{J_{n}}+\left(Q_{J_{n}}\right)^{*_{3}}=2\left(J_{n}+i \varepsilon J_{n+3}\right), \\
\left(J_{n}+i J_{n+1}\right)\left(Q_{J_{n}}\right)^{*_{4}}=\left(J_{2 n+1}-J_{n}^{2}\right)+\varepsilon\left(3 J_{n} J_{n+1}+J_{2 n+1}+2 J_{n+1}^{2}\right) \\
\quad+i \varepsilon(-1)^{n} 2^{n-1} \\
=\left(J_{n}-i J_{n+1}\right)\left(Q_{J_{n}}\right)^{*_{2}}, \\
\varepsilon Q_{J_{n}}+\left(Q_{J_{n}}\right)^{*_{5}}=J_{n+2}+i J_{n+3}, \\
Q_{J_{n}}-\varepsilon\left(Q_{J_{n}}\right)^{*_{5}}=J_{n}+i J_{n+1} .
\end{gathered}
$$

Proof. (3.17): By the Eq.(3.1) and (3.8) we get,

$$
Q_{J_{n}}\left(Q_{J_{n}}\right)^{*_{1}}=\left(J_{2 n+1}-J_{n}^{2}\right)+2 \varepsilon\left(3 J_{n} J_{n+1}+J_{2 n+1}+2 J_{n+1}^{2}\right) .
$$

(3.18): By the Eq.(3.1) and (3.9) we get,

$$
Q_{J_{n}}\left(Q_{J_{n}}\right)^{*_{2}}=\left(J_{n}^{2}-J_{n+1}^{2}\right)+2 i J_{n} J_{n+1} .
$$

(3.19): By the Eq.(3.1) and (3.10) we get,

$$
\begin{aligned}
Q_{J_{n}}\left(Q_{J_{n}}\right)^{*_{3}} & =\left(J_{n}^{2}+J_{n+1}^{2}\right)-2 i \varepsilon\left(J_{n+1} J_{n+2}-J_{n} J_{n+3}\right) \\
& =\left(J_{2 n+1}-J_{n}^{2}\right)-2 i \varepsilon(-1)^{n+1} 2^{n} .
\end{aligned}
$$


(3.20): By the Eq.(3.1) and (3.11) we get,

$$
Q_{J_{n}}\left(Q_{J_{n}}\right)^{*_{4}}=\left(J_{n}^{2}+J_{n+1}^{2}\right)=J_{2 n+1}-J_{n}^{2} .
$$

(3.21): By the Eq.(3.1) and (3.8) we get,

$$
Q_{J_{n}}+\left(Q_{J_{n}}\right)^{*_{1}}=2 J_{n}+2 \varepsilon J_{n+2}=2\left(J_{n}+\varepsilon J_{n+2}\right) .
$$

(3.22): By the Eq.(2.1) and (??) we get,

$$
Q_{J_{n}}+\left(Q_{J_{n}}\right)^{*_{2}}=2 J_{n}+2 i J_{n+1}=2\left(J_{n}+i J_{n+1}\right) .
$$

(3.23): By the Eq.(3.1) and (3.10) we get,

$$
Q_{J_{n}}+\left(Q_{J_{n}}\right)^{*_{3}}=2 J_{n}+2 i \varepsilon J_{n+3}=2\left(J_{n}+i \varepsilon J_{n+3}\right) .
$$

(3.24): By the Eq.(3.1), (3.9) and (3.11) we get,

$$
\begin{aligned}
\left(J_{n}+i J_{n+1}\right)\left(Q_{J_{n}}\right)^{*_{4}=}= & J_{n}^{2}+J_{n+1}^{2}-\varepsilon\left(J_{n}-i J_{n+1}\right)\left(J_{n+2}+i J_{n+3}\right) \\
= & J_{2 n+1}-J_{n}^{2}-\varepsilon\left[3 J_{n} J_{n+1}+J_{2 n+1}+2 J_{n+1}^{2}\right] \\
& +i \varepsilon(-1)^{n} 2^{n-1} \\
= & \left(J_{n}-i J_{n+1}\right)\left(Q_{J_{n}}\right)^{*_{2}} .
\end{aligned}
$$

(3.25): By the Eq.(3.1) and (3.12) we get,

$$
\varepsilon Q_{J_{n}}+\left(Q_{J_{n}}\right)^{* 5}=J_{n+2}+i J_{n+3} .
$$

(3.26): By the Eq.(3.1) and (3.12) we get,

$$
Q_{J_{n}}-\varepsilon\left(Q_{J_{n}}\right)^{* 5}=J_{n}+i J_{n+1} .
$$

In the following theorem, some properties related to the dual-complex Jacobsthal quaternions are given.

Theorem 3.2. Let $Q_{J_{n}}$ be the dual-complex Jacobsthal quaternion. In this case, we can give the following relations:

$$
\begin{aligned}
& Q_{J_{n+1}}+2 Q_{J_{n}}=Q_{J_{n+2}}, \\
& 2 Q_{J_{n+1}}-Q_{J_{n}}=Q_{j_{n}}, \\
& Q_{J_{n+1}}+2 Q_{J_{n-1}}=Q_{j_{n}} \\
& Q_{J_{n+1}}+2 Q_{J_{n}}=Q_{J_{n+2}}, \\
& 3\left(Q_{J_{n+2}}-Q_{J_{n-2}}\right)=Q_{j_{n+2}}-Q_{j_{n-2}}, \\
& \begin{aligned}
\left(Q_{J_{n+1}}\right)^{2}-4\left(Q_{J_{n-1}}\right)^{2}= & Q_{J_{2 n}}-J_{2 n+2}+i J_{2 n+1}+\varepsilon\left(J_{2 n+2}-2 J_{2 n+2}\right) \\
& +3 i \varepsilon J_{2 n+4},
\end{aligned} \\
& \begin{aligned}
\left(Q_{J_{n+1}}\right)^{2}+2\left(Q_{J_{n}}\right)^{2}= & Q_{J_{2 n+1}}-J_{2 n+3}+i J_{2 n+2}+\varepsilon\left(J_{2 n+3}+2 J_{2 n+5}\right) \\
& +3 i \varepsilon J_{2 n+4},
\end{aligned} \\
& Q_{J_{n}}-i\left(Q_{J_{n+1}}\right)^{*_{3}}-\varepsilon Q_{J_{n+2}}-i \varepsilon Q_{J_{n+3}}=J_{n}-J_{n+2}+2 \varepsilon J_{n+4} .
\end{aligned}
$$

Proof. It can be proved easily (3.27-3.31) by using (3.1).

(3.32):By the Eq.(3.1) we get,

$$
\begin{aligned}
\left(Q_{J_{n+1}}\right)^{2}-4\left(Q_{J_{n-1}}\right)^{2}= & \left(J_{2 n}-J_{2 n+2}\right)+2 i J_{2 n+1} \\
& +2 \varepsilon\left(J_{2 n+2}-J_{2 n+4}\right)+2 i \varepsilon\left(2 J_{2 n+3}\right) \\
= & J_{2 n}+i J_{2 n+1}+\varepsilon J_{2 n+2}+i \varepsilon J_{2 n+3} \\
& -J_{2 n+2}+i J_{2 n+1}+\varepsilon J_{2 n+4}+3 i \varepsilon J_{2 n+3} \\
= & Q_{J_{2 n}}-J_{2 n+2}+i J_{2 n+1}+\varepsilon\left(J_{2 n+2}-2 J_{2 n+4}\right) \\
& +i \varepsilon\left(3 J_{2 n+3}\right) .
\end{aligned}
$$


(3.33): By the Eq.(3.1) we get,

$$
\begin{aligned}
\left(Q_{J_{n+1}}\right)^{2}+2\left(Q_{J_{n}}\right)^{2}= & \left(J_{2 n+1}-J_{2 n+3}\right)+2 i J_{2 n+2} \\
& +2 \varepsilon\left(J_{2 n+3}-J_{2 n+5}\right)+2 i \varepsilon\left(2 J_{2 n+4}\right) \\
= & J_{2 n+1}+i J_{2 n+2}+\varepsilon J_{2 n+3}+i \varepsilon J_{2 n+4}-J_{2 n+3} \\
& +i J_{2 n+2}+\varepsilon\left(J_{2 n+3}+2 J_{2 n+5}\right)+3 i \varepsilon J_{2 n+4} \\
= & Q_{J_{2 n+1}}-J_{2 n+3}+i J_{2 n+2}+\varepsilon\left(J_{2 n+3}+2 J_{2 n+5}\right) \\
& +i \varepsilon\left(3 J_{2 n+4}\right) .
\end{aligned}
$$

(3.34): By the Eq.(3.10) we get,

$$
Q_{J_{n}}-i Q_{J_{n+1}^{*}}^{*_{3}}-\varepsilon Q_{J_{n+2}}-i \varepsilon Q_{J_{n+3}}=\left(J_{n}-J_{n+2}\right)+2 \varepsilon J_{n+4} .
$$

Theorem 3.3 (Honsberger identity). For $n, m \geq 0$ the Honsberger identity for the dual-complex Jacobsthal quaternions $Q_{J_{n}}$ and $Q_{J_{m}}$ is given by

$$
\begin{aligned}
2 Q_{J_{n}} Q_{J_{m}}+Q_{J_{n+1}} Q_{J_{m+1}}= & Q_{J_{n+m+1}}-J_{n+m+3}+i J_{n+m+2} \\
& +\varepsilon\left(J_{n+m+3}-2 J_{n+m+5}\right)+3 i \varepsilon J_{n+m+4} .
\end{aligned}
$$

Proof. (3.35): By the Eq.(3.1) we get,

$$
\begin{aligned}
2 Q_{J_{n}} Q_{J_{m}}+Q_{J_{n+1} Q_{J_{m+1}}=} & {\left.\left[2 J_{n} J_{m}+J_{n+1} J_{m+1}\right)-\left(2 J_{n+1} J_{m+1}+J_{n+2} J_{m+2}\right)\right] } \\
& +i\left[\left(2 J_{n} J_{m+1}+J_{n+1} J_{m+2}\right)+\left(2 J_{n+1} J_{m}+J_{n+2} J_{m+1}\right)\right] \\
& +\varepsilon\left[\left(2 J_{n} J_{m+2}+J_{n+1} J_{m+3}\right)-\left(2 J_{n+1} J_{m+3}+J_{n+2} J_{m+4}\right)\right. \\
& \left.+\left(2 J_{n+2} J_{m}+J_{n+3} J_{m+1}\right)-\left(2 J_{n+3} J_{m+1}+J_{n+4} J_{m+2}\right)\right] \\
& +i \varepsilon\left[\left(2 J_{n} J_{m+3}+J_{n+1} J_{m+4}\right)+\left(2 J_{n+1} J_{m+2}+J_{n+2} J_{m+3}\right)\right. \\
& \left.+\left(2 J_{n+2} J_{m}+J_{n+3} J_{m+1}\right)+\left(2 J_{n+3} J_{m+1}+J_{n+4} J_{m+2}\right)\right] \\
= & \left(J_{n+m+1}-J_{n+m+3}\right)+2 i J_{n+m+2} \\
& +2 \varepsilon\left(J_{n+m+3}-J_{n+m+5}\right)+4 i \varepsilon\left(J_{n+m+4}\right) \\
= & \left(J_{n+m+1}+i J_{n+m+2}+\varepsilon J_{n+m+3}+i \varepsilon J_{n+m+4}\right) \\
& -J_{n+m+3}+i J_{n+m+2}+\varepsilon\left(J_{n+m+3}-2 J_{n+m+5}\right) \\
& +3 i \varepsilon J_{n+m+4} \\
= & Q J_{n+m+1}-J_{n+m+3}+i J_{n+m+2}+\varepsilon\left(J_{n+m+3}-2 J_{n+m+5}\right) \\
& +3 i \varepsilon J_{n+m+4} .
\end{aligned}
$$

where the identity $J_{n} J_{m+1}+2 J_{n-1} J_{m}=J_{n+m}$ was used [19]. (Table 3)

Table 3. Types of Jacobsthal quaternions

\begin{tabular}{cc}
\hline & Honsberger identity (1985) \\
\hline Jacobsthal Number & $J_{n+1} J_{m+1}+2 J_{n} J_{m}=J_{n+m+1}$ \\
\hline Jacobsthal Quaternions & $J Q_{n} J Q_{m+2} J Q_{n-1} Q_{m-1}=2 J Q_{n+m+1}-J_{n+m-1}-J_{n+m+1}-J_{n+m+3}-J_{n+m+5}$ \\
\hline k-Jacobsthal Quaternions & $D_{n}^{J} D_{m}^{J}+2 D_{n-1}^{J} D_{m-1}^{J}=2 D_{n+m-1}^{J}-J_{n+m-1}$ \\
\hline $\begin{array}{c}\text { Dual Jacobsthal Quaternions } \\
\text { Quaternions }\end{array}$ & $Q_{J n+1} Q_{J m+1}+2 Q_{J n} Q_{J m}=Q_{J n+m+1}-J_{n+m+3}+i J_{n+m+2}+\varepsilon\left(J_{n+m+3}-2 J_{n+m+5}\right)$ \\
\hline $\begin{array}{c}\text { Dual-complex Jacobsthal } \\
n+m+4\end{array}$ \\
\hline
\end{tabular}

Theorem 3.4 (D'ocagne's identity). For $n, m \geq 0$ the D'ocagne's identity for the dual-complex Jacobsthal quaternions $Q_{J_{n}}$ and $Q_{J_{m}}$ is given by

$$
Q_{J_{m}} Q_{J_{n+1}}-Q_{J_{m+1}} Q_{J_{n}}=(-1)^{n} 2^{n} J_{m-n}(3+i+15 \varepsilon+5 i \varepsilon) .
$$


Proof. (3.36): By the Eq.(3.1) we get,

$$
\begin{aligned}
Q_{J_{m}} Q_{J_{n+1}}-Q_{J_{m+1}} Q_{J_{n}}= & {\left[\left(J_{m} J_{n+1}-J_{m+1} J_{n}\right)-\left(J_{m+1} J_{n+2}-J_{m+2} J_{n+1}\right)\right] } \\
& +i\left[\left(J_{m} J_{n+2}-J_{m+1} J_{n+1}\right)+\left(J_{m+1} J_{n+1}-J_{m+2} J_{n}\right)\right] \\
& +\varepsilon\left[\left(J_{m} J_{n+3}-J_{m+1} J_{n+2}\right)+\left(J_{m+2} J_{n+1}-J_{m+3} J_{n}\right)\right. \\
& \left.-\left(J_{m+1} J_{n+4}-J_{m+2} J_{n+3}\right)-\left(J_{m+3} J_{n+2}-J_{m+4} J_{n+1}\right)\right] \\
& +i \varepsilon\left[\left(J_{m} J_{n+4}-J_{m+1} J_{n+3}\right)+\left(J_{m+1} J_{n+3}-J_{m+2} J_{n+2}\right)\right. \\
& \left.+\left(J_{m+2} J_{n+2}-J_{m+3} J_{n+1}\right)+\left(J_{m+3} J_{n+1}-J_{m+4} J_{n}\right)\right] \\
= & (-1)^{n} 2^{n} J_{m-n}(3+i+15 \varepsilon+5 i \varepsilon) .
\end{aligned}
$$

where the identity $J_{m} J_{n-1}-J_{m-1} J_{n}=(-1)^{n} 2^{n-1} J_{m-n}$ was used [19].

Theorem 3.5. Let $Q_{J_{n}}$ be the dual-complex Jacobsthal quaternion.Then, we have the following identities

$$
\begin{gathered}
\sum_{s=1}^{n} Q_{J_{s}}=\frac{1}{2}\left[Q_{J_{n+2}}-Q_{J_{2}}\right], \\
\sum_{s=0}^{p} Q_{J_{n+s}}=\frac{1}{2}\left[Q_{J_{n+p+2}}-Q_{J_{n+1}}\right], \\
\sum_{s=1}^{n} Q_{J_{2 s-1}}=\frac{2}{3} Q_{J_{2 n}}-\frac{1}{3}\left[n\left(2 Q_{J_{2}}-Q_{J_{3}}\right)-2 Q_{J_{0}}\right], \\
\sum_{s=1}^{n} Q_{J_{2 s}}=\frac{2}{3} Q_{J_{2 n+1}}-\frac{1}{3}\left[n\left(2 Q_{J_{2}}-Q_{J_{3}}\right)+2 Q_{J_{1}}\right] .
\end{gathered}
$$

Proof. (3.37) Since $\sum_{s=a}^{n} J_{s}=J_{n+2}-J_{a+1}[3]$, we get

$$
\begin{aligned}
\sum_{s=1}^{n} & Q_{J_{s}}=\sum_{s=1}^{n} J_{s}+i \sum_{s=1}^{n} J_{s+1}+\varepsilon \sum_{s=1}^{n} J_{s+2}+i \varepsilon \sum_{s=1}^{n} J_{s+3} \\
& =\frac{1}{2}\left[\left(J_{n+2}-J_{2}\right)+i\left(J_{n+3}-J_{3}\right)+\varepsilon\left(J_{n+4}-J_{4}\right)+i \varepsilon\left(J_{n+5}-J_{5}\right)\right] \\
& =\frac{1}{2}\left[\left(J_{n+2}+i J_{n+3}+\varepsilon J_{n+4}+i \varepsilon J_{n+5}\right)-\left(J_{2}+i J_{3}+\varepsilon J_{4}+i \varepsilon J_{5}\right)\right] \\
& =\frac{1}{2}\left[Q_{J_{n+2}}-Q_{J_{2}}\right] .
\end{aligned}
$$

(3.38): Hence, we can write

$$
\begin{aligned}
\sum_{s=0}^{p} Q_{J_{n+s}=} & \frac{1}{2}\left[\left(J_{n+p+2}-J_{n+1}\right)+i\left(J_{n+p+3}-J_{n+2}\right)\right. \\
& \left.+\varepsilon\left(J_{n+p+4}-J_{n+3}\right)+i \varepsilon\left(J_{n+p+5}-J_{n+4}\right)\right] \\
= & \frac{1}{2}\left[\left(J_{n+p+2}+i J_{n+p+3}+\varepsilon J_{n+p+4}+i \varepsilon J_{n+p+5}\right)\right. \\
& \left.-\left(J_{n+1}+i J_{n+2}+\varepsilon J_{n+3}+i \varepsilon J_{n+4}\right)\right] \\
= & \frac{1}{2}\left[Q_{J_{n+p+2}}-Q_{J_{n+1}}\right] .
\end{aligned}
$$

(3.39): Using $\sum_{i=0}^{n-1} J_{2 i+1}=\frac{1}{3}\left(2 J_{2 n}+n\right)$ and $\sum_{i=0}^{n} J_{2 i}=\frac{1}{3}\left(2 J_{2 n+1}-n-2\right)$ [21, 22], we get

$$
\begin{aligned}
\sum_{s=1}^{n} Q_{J_{2 s-1}=} & \frac{1}{3}\left[\left(2 J_{2 n}+n\right)+i\left(2 J_{2 n+1}-n-2\right)\right. \\
& \left.+\varepsilon\left(2 J_{2 n+2}+n-2\right)+i \varepsilon\left(2 J_{2 n+3}-n-6\right)\right] \\
= & \frac{2}{3}\left[J_{2 n}+i J_{2 n+1}+\varepsilon J_{2 n+2}+i \varepsilon J_{2 n+3}\right] \\
& +\frac{1}{3}[n(1-i+\varepsilon-i \varepsilon)-2(i+\varepsilon+3 i \varepsilon)] \\
= & \frac{2}{3} Q_{J_{2 n}}+\frac{1}{3}\left[n\left(2 Q_{J_{2}}-Q_{J_{3}}\right)-2 Q_{J_{0}}\right] .
\end{aligned}
$$


(3.40): Using $\sum_{i=0}^{n} J_{2 i}=\frac{1}{3}\left(J_{2 n+1}-n-2\right) \quad[21,22]$, we obtain

$$
\begin{aligned}
\sum_{s=1}^{n} Q_{J_{2 s}=} & \frac{1}{3}\left[\left(2 J_{2 n+1}-n-2\right)+i\left(2 J_{2 n+2}+n-2\right)\right. \\
& \left.+\varepsilon\left(2 J_{2 n+3}-n-6\right)+i \varepsilon\left(2 J_{2 n+4}+n-10\right)\right] \\
= & \frac{2}{3}\left[J_{2 n+1}+i J_{2 n+2}+\varepsilon J_{2 n+3}+i+\varepsilon J_{2 n+4}\right] \\
& +\frac{1}{3}[-n(1-i+\varepsilon-i \varepsilon)-2(1+i+3 \varepsilon+5 i \varepsilon)] \\
= & \frac{2}{3} Q_{J_{2 n+1}}-\frac{1}{3}\left[n\left(2 Q_{J_{2}}-Q_{J_{3}}\right)+2 Q_{J_{1}}\right] .
\end{aligned}
$$

Theorem 3.6 (Binet's Formula). Let $Q_{J_{n}}$ be the dual-complex Jacobsthal quaternion. For $n \geq 1$, Binet's formula for these quaternions is as follows:

$$
Q_{J_{n}}=\frac{1}{\alpha-\beta}\left(\hat{\alpha} \alpha^{n}-\hat{\beta} \beta^{n}\right)
$$

where

$$
\hat{\alpha}=1+i \alpha+\varepsilon \alpha^{2}+i \varepsilon \alpha^{3}, \quad \alpha=2
$$

and

$$
\hat{\beta}=1+i \beta+\varepsilon \beta^{2}+i \varepsilon \beta^{3}, \quad \beta=-1 .
$$

Proof. (3.41): By the Binet's formula of Jacobsthal sequence [3] we get,

$$
\begin{aligned}
Q_{J_{n}} & =\frac{\alpha^{n}-\beta^{n}}{3}+i \frac{\alpha^{n+1}-\beta^{n+1}}{3}+\varepsilon \frac{\alpha^{n+2}-\beta^{n+2}}{3}+i \varepsilon \frac{\alpha^{n+3}-\beta^{n+3}}{3} \\
& =\frac{\alpha^{n}\left(1+i \alpha+\varepsilon \alpha^{2}+i \varepsilon \alpha^{3}\right)-\beta^{n}\left(1+i \beta+\varepsilon \beta^{2}+i \varepsilon \beta^{3}\right)}{3} \\
& =\frac{1}{3}\left(\hat{\alpha} \alpha^{n}-\hat{\beta} \beta^{n}\right) .
\end{aligned}
$$

Binet's formula of the dual-complex Jacobsthal quaternion is the same as Binet's formula of the Jacobsthal quaternion [3].

Theorem 3.7 (Cassini's Identity). Let $Q_{J_{n}}$ be the dual-complex Jacobsthal quaternion. For $n \geq 1$, Cassini's identity for $Q_{J_{n}}$ is as follows:

$$
Q_{J_{n-1}} Q_{J_{n+1}}-Q_{J_{n}}^{2}=(-1)^{n} 2^{n-1}(3+i+15 \varepsilon+5 i \varepsilon) .
$$

Proof. (3.42): By using (3.1) we get

$$
\begin{aligned}
& Q_{J_{n+1}} Q_{J_{n-1}}-\left(Q_{J_{n}}\right)^{2}= {\left[\left(J_{n+1} J_{n-1}-J_{n}^{2}\right)-\left(J_{n+2} J_{n}-J_{n+1}^{2}\right)\right] } \\
&+i\left[\left(J_{n+1} J_{n}-J_{n} J_{n+1}\right)+\left(J_{n+2} J_{n-1}-J_{n+1} J_{n}\right)\right] \\
&+\varepsilon\left[\left(J_{n+1} J_{n+1}-J_{n} J_{n+2}\right)+\left(J_{n+3} J_{n-1}-J_{n+2} J_{n}\right)\right. \\
&-\left(J_{n+2} J_{n+2}-J_{n+1} J_{n+3}\right) \\
&+\left.-\left(J_{n+4} J_{n}-J_{n+3} J_{n+1}\right)\right] \\
&+ {\left[\left(J_{n+1} J_{n+2}-J_{n} J_{n+3}\right)\right.} \\
&-\left(J_{n+3} J_{n}-J_{n+2} J_{n+1}\right) \\
&+\left(J_{n+3} J_{n}-J_{n+2} J_{n+1}\right) \\
&\left.+\left(J_{n+4} J_{n-1}-J_{n+3} J_{n}\right)\right] \\
&=(-1)^{n} 2^{n-1}(3+i+15 \varepsilon+5 i \varepsilon) .
\end{aligned}
$$

where the identities of the Jacobsthal numbers $J_{m} J_{n+1}-J_{m+1} J_{n}=(-1)^{n} 2^{n-1} J_{m-n}$ and $J_{n+1} J_{n-1}-J_{n}{ }^{2}=$ $(-1)^{n} 2^{n-1}$ are used [3] (Table 4).

Theorem 3.8 (Catalan's Identity). Let $Q_{J_{n}}$ be the dual-complex Jacobsthal quaternion. For $n \geq 1$, Catalan's identity for $Q_{J_{n}}$ is as follows:

$$
Q_{J_{n+r}} Q_{J_{n-r}}-\left(Q_{J_{n}}\right)^{2}=(-1)^{n-r+1} 2^{n-r} J_{r}^{2}(3+i+15 \varepsilon+5 i \varepsilon) .
$$


Table 4. Types of Jacobsthal quaternions

\begin{tabular}{cc}
\hline & Cassini's identity \\
\hline Jacobsthal number & $J_{n+1} J_{n-1}-J_{n}^{2}=(-1)^{n} 2^{n-1} J_{1}$ \\
\hline Jacobsthal quaternions & $J Q_{n-1} J Q_{n+1}-J Q_{n}^{2}=(-1)^{n} 2^{n-1}(7+5 i+7 j+5 k)$ \\
\hline k-Jacobsthal quaternions & $Q_{J k, n-1} Q_{J k, n+1}-Q_{J k, n}^{2}=(\alpha \beta)(-2)^{n-1}$ \\
\hline $\begin{array}{c}\text { Dual Jacobsthal quaternions } \\
\text { Dual-complex Jacobsthal } \\
\text { quaternions }\end{array}$ & $D_{n-1}^{J} D_{n+1}^{J}-\left(D_{n}^{J}\right)^{2}=(-1)^{n} 2^{n-1}(1+i+5 j+7 k)$ \\
\hline
\end{tabular}

Proof. (3.43): By using (3.1) we get,

$$
\begin{aligned}
Q_{J_{n+r}} Q_{J_{n-r}}-\left(Q_{J_{n}}\right)^{2}= & {\left[\left(J_{n+r} J_{n-r}-J_{n}^{2}\right)-\left(J_{n+r+1} J_{n-r+1}-J_{n+1}^{2}\right)\right] } \\
+ & i\left[\left(J_{n+r} J_{n-r+1}-J_{n} J_{n+1}\right)\right. \\
& \left.+\left(J_{n+r+1} J_{n-r}-J_{n+1} J_{n}\right)\right] \\
+\varepsilon[ & \left(J_{n+r} J_{n-r+2}-J_{n} J_{n+2}\right) \\
& +\left(J_{n+r+2} J_{n-r}-J_{n+2} J_{n}\right) \\
& -\left(J_{n+r+1} J_{n-r+3}-J_{n+1} J_{n+3}\right) \\
& \left.-\left(J_{n+r+3} J_{n-r+1}-J_{n+3} J_{n+1}\right)\right] \\
+i \varepsilon\left[\left(J_{n+r} J_{n-r+3}-J_{n} J_{n+3}\right)\right. & +\left(J_{n+r+3} J_{n-r}-J_{n+3} J_{n}\right) \\
& +\left(J_{n+r+1} J_{n-r+2}-J_{n+1} J_{n+2}\right) \\
& \left.+\left(J_{n+r+2} J_{n-r+1}-J_{n+2} J_{n+1}\right)\right] \\
= & -2)^{n-r} J_{r}^{2}(3+i+15 \varepsilon+5 i \varepsilon) .
\end{aligned}
$$

where the identities of the Jacobsthal numbers $J_{m} J_{n-1}-J_{m-1} J_{n}=(-1)^{n} 2^{n-1} J_{m-n}$ [19] and $J_{n+r} J_{n-r}-J_{n}^{2}=$ $-(-2)^{n-r} J_{r}^{2}$ are used.

Table 5. Types of Jacobsthal quaternions

\begin{tabular}{cc}
\hline & Catalan's identity \\
\hline Jacobsthal number & $J_{n+r} J_{n-r}-J_{n}^{2}=-(-2)^{n-r} J_{r}^{2}$ \\
\hline Jacobsthal quaternions & $J Q_{n-r} J Q_{n+r}-J Q_{n}^{2}=-(-2)^{n-r} J_{r}^{2}(7+5 i+7 j+5 k)$ \\
\hline k-Jacobsthal quaternions & $Q_{J k, n-r} Q_{J k, n+r}-Q_{J}{ }_{k, n}^{2}=(-1)^{n-r+1}(-2)^{n-1} J_{k, r}^{2}$ \\
\hline Dual Jacobsthal quaternions & $D_{n-r}^{J} D_{n+r}^{J}-\left(D_{n}^{J}\right)^{2}=-(-2)^{n-r} J_{r}^{2}(1+i+5 j+7 k)$ \\
\hline $\begin{array}{c}\text { Dual-complex Jacobsthal } \\
\text { quaternions }\end{array}$ & $Q_{J n+r} Q_{J n-r}-Q_{J}{ }_{n}^{2}=-(-2)^{n-r} J_{r}^{2}(3+i+15 \varepsilon+5 i \varepsilon)$ \\
\hline
\end{tabular}

\section{Conclusions}

In this study, a number of new results on dual-complex Jacobsthal quaternions were derived. Quaternions have great importance as they are used in quantum physics, applied mathematics, quantum mechanics, Lie groups, kinematics and differential equations.

This study fills the gap in the literature by providing the dual-complex Jacobsthal quaternion using definitions of the dual-complex number [10], dual-complex Fibonacci number [11] and Jacobsthal quaternions [20] and [21].

\section{References}

[1] Hamilton W. R.: Elements of Quaternions. Longmans, Green and Co., London, (1866).

[2] Sloane N. J. A.: A Handbook of Integer Sequences. New York, Press, (1973).

[3] Horadam A. F.: Jacobsthal Representation Numbers. The Fibonacci Quarterly. 34, 40-54 (1996). 
[4] Horadam A. F.: Jacobsthal Representation Polynomials. The Fibonacci Quarterly. 35, 137-148 (1997).

[5] Clifford W. K.: A preliminary sketch of biquaternions, (1873).

[6] Majernik, V.: Quaternion formulation of the Galilean space-time transformation. Acta Phy. Slovaca. 56 (1), 9-14 (2006).

[7] Kotelnikov, A. P.: Screw calculus and some of its applications to geometry and mechanics. Annals of Imperial University of Kazan (1895).

[8] Study, E.: Geometrie der Dynamen. Leipzig. (1903).

[9] Majernik, V.: Multicomponent number systems. Acta Pyhsica Polonica A. 90 (3), 491-498 (1996).

[10] Messelmi, F.: Dual -complex numbers and their holomorphic functions. https://hal.archives-ouvertes.fr/hal01114178, (2015).

[11] Gungor, M. A., Azak, A. Z.: Investigation of Dual-Complex Fibonacci, Dual-Complex Lucas Numbers and Their Properties. Advances in Applied Clifford Algebras. 27 (4), 3083-3096 (2017).

[12] Djordjevid G. B.: Generalized Jacobsthal Polynomials. The Fibonacci Quarterly, 38 (3), 239-243 (2009).

[13] Djordjevid G. B.: Derivative sequences of generalized Jacobsthal and Jacobsthal-Lucas polynomials. Fibonacci Quarterly. 38 (4), 334-338 (2000).

[14] Cerin Z.: Sums of Squares and Products of Jacobsthal Numbers. Journal of Integer Sequence. 10, Article 07.2.5, (2007).

[15] Cerin Z.: Formulae for Sums of Jacobsthal-Lucas Numbers. International Mathematical Forum. 2 (40), 1969-1984 (2007).

[16] Köken F., Bozkurt D.: On the Jacobsthal Numbers by Matrix Methods. International Journal of Contemporary Mathematical Sciences. 3 (13), 605-614 (2008).

[17] Köken F., Bozkurt D.: On the Jacobsthal-Lucas Numbers by Matrix Methods. International Journal of Contemporary Mathematical Sciences. 3 (13), 1629-1633 (2008).

[18] Daşdemir A.: On the Jacobsthal Numbers by Matrix Method. SDU Journal of Science (E-Journal). 71, 69-76 (2012).

[19] Daşdemir A.: A study on the Jacobsthal and Jacobsthal -Lucas numbers by matrix method. DUFED Journal of Sciences 3, (1), 13-18 (2014).

[20] Szynal-Liana A., Włoch I.: A Note on Jacobsthal Quaternions. Advances in Applied Clifford Algebras 26 (1), 441-447 (2016).

[21] Aydın Torunbalcı F., Yüce, S.: A New Approach to Jacobsthal Quaternions. Filomat 31 (18), 5567-5579 (2017).

[22] Taşçı D.: On k-jacobsthal and k-jacobsthal-Lucas quaternions. Journal of Science and Arts 3 469-476 (2017).

[23] Aydın, Torunbalc1 F.: Dual Jacobstthal quaternions. Communication in advanced Mathamatical Sciences. 3 (3), 130-142 (2020).

\section{Affiliations}

\section{FÜGEN TORUNBALCI AYDIN}

ADDRESS: Yildiz Technical University

Faculty of Chemical and Metallurgical Engineering, Department of Mathematical Engineering

34220

Istanbul

Turkey.

E-MAIL: faydin@yildiz.edu.tr; ftorunay@gmail.com

ORCID ID:0000-0001-9292-1832 